\title{
Endoscopic Recording (or its Absence): A Disappointment
}

\author{
Robert J Shprintzen ${ }^{1 *}$ and Pablo Antonio Ysunza ${ }^{2}$ \\ ${ }^{1}$ President and Chairman of the Board, The Virtual Center for Velo-Cardio-Facial Syndrome, USA \\ ${ }^{2}$ Ian Jackson Craniofacial and Cleft Palate Clinic of Beaumont Hospital Royal Oak, Michigan, Professor of Physical Medicine and \\ Rehabilitation, Oakland University, William Beaumont School of Medicine, USA
}

*Corresponding author: Robert J Shprintzen, President and Chairman of the Board, The Virtual Center for Velo-Cardio-Facial Syndrome, USA

\section{Short Communication}

One of the advantages of being old and seeing science and clinical practice advance is that as time passes, we can observe those things that succeed and those that fail if we are perfectly honest in our assessments. Moreover, we can determine if practices we thought were transferable to our colleagues simply by describing them in the literature have often proven to be false hopes that leave us scratching our heads in order to determine just what went wrong. Such is the case with endoscopic procedures designed to assess the upper airway anatomy and physiology [1]. Between the two of us, we have more than 75 years of combined experience with flexible fiberoptic nasopharyngoscopy of the upper airway, literally thousands of cases examined both individually and together, and between us, we have published approximately 200 articles, chapters and books that directly or indirectly report on the use of flexible fiberoptic nasopharyngoscopy in people with upper airway disorders, respiratory obstruction, speech disorders, and voice disorders. In other words, time has afforded us the opportunity to see the application of endoscopic procedures from their beginnings to the current state of the art practice $[2,3]$. What we see disturbs us mightily.

The first author was introduced to endoscopic procedures for assessing velopharyngeal insufficiency in 1974 when he met and became familiar with the work of two pioneers in imaging procedures who were on opposite ends of the planet, specifically the United Kingdom and Japan. Ron Pigott, a plastic surgeon at Frenchay Hospital in Bristol, UK, and Tadashi Miyazaki, head of the Department of Maxillofacial Surgery at Osaka University Dental School in Japan published and presented papers in the late 1960s and early 1970s describing the application of nasopharyngoscopy to assess velopharyngeal closure [4]. Mr. Pigott published papers describing the use of both flexible fiberoptic endoscopes and rigid endoscopes in 1969. Dr. Miyazaki and his colleagues, Drs. Matsuya and Yamaoka, described the use of a side-viewing flexible fiberoptic endoscope of what at that time was a narrow diameter just above $3 \mathrm{~mm}$. We were introduced to narrow diameter endviewing pediatric fiberoptic endoscopes several years later and immediately applied them in day-to-day clinical use and research, both for children and adults. With flexible nasopharyngoscopy applicable to assessing speech, voice, respiration, and swallowing being available in the Americas since the 1970s, the market for the instruments expanded dramatically and smaller, narrow diameter endoscopes with improved optics and light transmission characteristics were rapidly developed by a number of companies. The introduction of recording the examinations with video were introduced by both the team in Osaka and Mr. Pigott in Bristol nearly simultaneously. In our own institutions, the ability to record the examinations and share them with our interdisciplinary cleft palate teams advanced our treatment techniques dramatically and increased a transdisciplinary appreciation for the complexity of normal speech production.

Today, flexible fiberoptic nasopharyngolaryngoscopes are ubiquitous in the offices of otolaryngologists worldwide. High definition video/sound recordings are easy to accomplish and should be inexpensive and simple, whether equipment is purchased as a package from commercial medical instrument companies or rigged by clinicians who have some knowledge of digital recording of endoscopic images. The process is not difficult. Because the information is used often in the assessment of speech and vocal production, the recording of speech synchronous to the video recording is essential. Data storage is inexpensive with digital recording devices storing four terabytes of information available for as little as $\$ 100$. Four terabytes provide even busy clinicians enough storage space for years. Nonetheless, many clinicians do not record office endoscopies, or they record video without sound thereby rendering later review impossible. Modern computer equipment is the perfect platform for recording endoscopic studies with sound and high quality (high definition video with 1080p or higher resolution) digital images. The first author on this paper put 
together his own system consisting of a Mac computer (any Mac computer would do) with video recording software (lowest price would be approximately 800 total, an endoscopic video camera (available for approximately $\$ 1500$ and higher), a microphone (\$50) and digital storage (\$100). Thus, an entire system to allow recording and storage of digital video and sound would be well under $\$ 3,000$, the cost of which would be amortized within a very short period.

Why would it be essential to record endoscopic examinations? That the question has to be asked is disappointing. Let's develop an imaginary scenario. You, the reader, have a complaint of difficulty catching your breath, coughing frequently, a fever, and malaise. The doctor who sees you thinks that you may have pneumonia, so the doctor orders a radiographic procedure to look at your lungs. The doctor does a fluoroscopy but does not record it or print a picture from the study. You go to a second doctor who believes you simply have a severe allergic reaction to pollen and wonders why the other doctor thought you had pneumonia. The second asks the first doctor for copies of the x-ray study. The first doctor says, "I didn't put it on film, but I saw evidence of pneumonia." The second doctor indicates his displeasure because the first doctor's findings disagree with the second doctor's. Would you trust a verbal report of a radiographic procedure that only one person has seen? A rhetorical question, we hope. Moreover, the interpretation of endoscopic studies is variable leading to possible false positive/negative findings affecting treatment recommendations. If not recorded, the value of the study is questionable. The authors reviewed 1,525 consecutive nasopharyngoscopic examinations with recorded video and sound resulted in 598 surgical recommendations including velopharyngeal reconstruction, adenoidectomy, and/ or tonsillectomy and surgical airway management. In all cases, surgery was based on review of recorded studies by the surgeon and endoscopist, and often by other clinicians and students. In a subset of 200 consecutive reviewed by three or more clinicians, we found that interpretation disagreements occurred in $48 \%$ of cases. Disagreements were relevant to treatment decisions in 94\%. Group reviews eventually resulted in consensus agreement. Efficacy was measured by surgical outcomes for velopharyngeal insufficiency and obstructive airway procedures (94\% and 95\% respectively). Because we currently consult on cases from the U.S. and overseas, we often request copies of endoscopic videos; 296 requests have been made since 2012, 221 from the US, 75 from Europe Of these, $48 \%$ from the U.S. and $39 \%$ from overseas had available video recordings with less than one-third of the videos having sound [5]. The inability to review completed studies may lead to treatment error, unacceptable for any imaging procedure. It is time that clinicians stop doing imaging procedures that cannot be reviewed by more than one person. An endoscopic study without video and sound recording is archaic, inadequate, and not to be trusted.

\section{References}

1. Matsuya T, Miyazaki T, Yamaoka M (1974) Fiberscopic examination of velopharyngeal closure in normal individuals. Cleft Palate J 11: 286-291.

2. Miyazaki M, Matsuya T, Yamaoka M (1975) Fiberscopic methods for assessment of velopharyngeal closure during various activities. Cleft Palate J 12: 107-114.

3. Pigott RW (1969) The nasendoscopic appearance of the normal palatopharyngeal valve. Plast Reconst. Surg 43: 19-24.

4. Pigott RW, Benson JF, White FP (1969) Nasendoscopy in the diagnosis of velopharyngeal incompetence. Plas Reconstr Surg 43: 141-147.

5. Shprintzen RJ, Ysunza PA (2017) What did you see? What did you hear? Unrecorded endoscopic examinations. Paper presented at the annual meeting of The Society for Ear, Nose, and Throat Advances in Children (SENTAC), Toronto, USA.
This work is licensed under Creative Commons Attribution 4.0 License

To Submit Your Article Click Here:

Submit Article
DOI: $10.32474 /$ SJ0.2019.02.000149

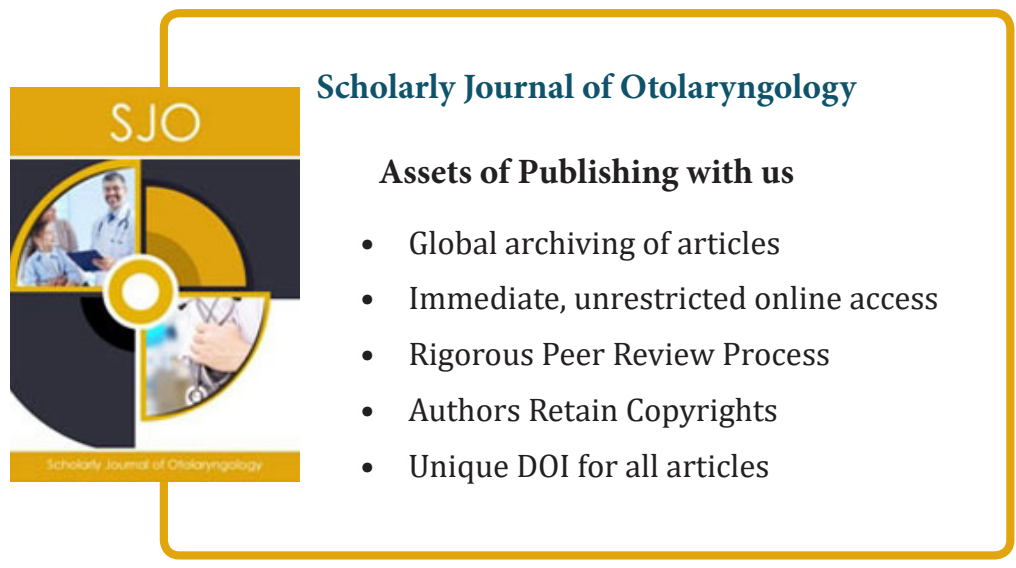

